\title{
Comparative Analysis of the Dynamics of Heart Rhythm Variability Indicators of Long-Distance Runners in Different Training Conditions
}

\author{
Vladislav Bakayev ${ }^{1}$ and Alexander Bolotin ${ }^{1}$ \\ 'Peter the Great St. Petersburg Polytechnic University, Institute of Physical Education, Sports and Tourism, St. Petersburg, Russia
}

\begin{abstract}
It has been established that the dynamics of rest/load indicators, as a rule, depends on the skill level of the athlete. More successful athletes have a high level of sympathetic reaction and activity of the central circuit for the regulation of heart rhythm to the load as a manifestation of functional reserves mobilization. A healthy powerful reaction to load and a high level of mobilization abilities are the most important parameters for the success of training and competitive activities during race running. The findings of a comprehensive assessment of heart rate variability in the evaluation of short 5-minute records and long (weekly) records indicate that, compared with athletes who train at the medium altitude, long-distance runners training on the flat terrain adapt to the conditions of sports activity with higher strain on regulatory systems. This is due to the lack of proper economization against the background of the initially low reserve and aerobic capabilities of the body, due, inter alia, to the low development of the autonomous control circuit. The above leads to overload and depreciation of the central control circuit at rest and, in connection with this, its low response to the impact under the conditions of physical and competitive loads. The cost of adaptation of such an organism increases significantly, its adaptation and competitive potential decreases. Accordingly, with a highly developed autonomous control circuit during training at the medium altitude, we can expect a higher competitive success of the long-distance runners compared to the long-distance runners who train on the flat terrain with poorly expressed control circuit.
\end{abstract}

Key words: heart rate variability indicators, long-distance runners, stayers, training at the medium altitude, stamina development

\section{Introduction}

Today, the practice of training long-distance runners creates constantly increasing requirements for the body of athletes. At the same time, scientific approaches to the preparation of long-distance runners remain poorly developed, which largely limits the possibility of effective use of training tools and methods (Bakaev, Bolotin, \& Vasileva, 2015; Bohuslavska, Furman, Pityn, Galan, \& Nakonechnyi, 2017; Bolotin, \& Bakayev, 2017a; Dong, 2016).
In the course of adaptation of the long-distance runners' bodies to the conditions of training and competition, all the life support mechanisms of the body are involved. Moreover, the training of long-distance runners on the flat terrain and at the medium altitude have different characteristics (Bolotin, \& Bakayev, 2017b; Bakaev, Bolotin, \& Aganov, 2016; Bakayev, Bolotin, \& You, 2018). This affects the formation of the structural trace of adaptation, which must be considered in the training of these athletes (Bolotin, \& Bakayev, 2017c;

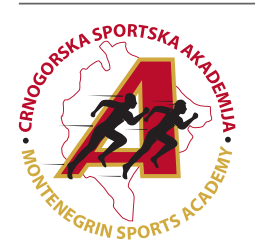

Correspondence: 
Boullosa, Tuimil, Leicht, \& Crespo-Salgado, 2009; Dong, 2016; Bolotin, \& Bakayev, 2018; Bolotin, \& Bakayev, 2016).

The purpose of the study is to conduct a comparative analysis of the nature of changes in the dynamics of heart rate variability in long-distance runners during training under the conditions of medium altitude and on flat terrain.

\section{Study Objectives}

1) Evaluate the initial level of heart rate variability in long-distance runners before training under the conditions of medium altitude and on flat terrain.

2) Investigate changes in heart rate variability in long-distance runners under the influence of physical exertion at medium altitude and on flat terrain.

3) Identify the nature of recovery after the training load of the long-distance runners on the basis of changes in their heart rate variability.

\section{Methods}

In the course of the study, two groups of long-distance runners with seven athletes in each group were examined. The first group (EG-1) trained under the conditions of the flat terrain, and the second group (EG-2) trained under conditions of medium altitude at an altitude of 2,100 meters. Studies were conducted at the training camps during their preparation for the competition. The level of sports preparedness of long-distance runners in both groups was the same. Their heart rate variability was examined before the commencement. Then they were examined 35 days later, after exposure to training loads under the conditions of medium altitude and on flat terrain. Five days before the start of the competition, we conducted comparative analysis of changes in heart rate variability in long-distance runners under the influence of physical exertion at medium altitude and on flat terrain. We assessed the dynamics in changes in heart rate variability under the influence of training loads at medium altitude and on flat terrain.

\section{The tasks were solved by using the following methods}

1) Theoretical analysis and synthesis of scientific and methodological and special literature, documentary materials and long-distance runners training programs, generalization of modern technologies of their sports training.

2) The pedagogical experiment was multi-level, natural, was ascertaining and comparative in nature and consisted of two series:

- comparative assessment of long-distance runners training on the flat terrain and at medium altitude, as well as of their recovery;

- comparative analysis of the dynamics of heart rhythm variability indicators of long-distance runners during training under the conditions of medium altitude and on flat terrain.

3) Mathematical and statistical analysis of empirical data.

To assess the athletes' adaptation to the conditions of training loads under the conditions of medium altitude and on flat terrain, as well as to assess the regulatory and compensatory mechanisms, the study used the analysis of pulsometry and heart rate variability (HRV) of athletes using rhythmocardiography (RCG).

It was taken into account that if long-distance runners do not have enough functional reserves, then adaptive regula- tory systems function intensely. On the RCG, this is manifested in a high degree of the heart rhythm stabilization. That is, the heartbeat is characterized by pronounced regularity like a metronome. At the same time, the normal heart rhythm is normally characterized by irregularity, and its degree reflects the adaptation potential of the body to a certain extent. This is due to the predominance of the central or autonomous circuit of heart rhythm regulation by the regulatory systems of the body of the long-distance runners. In addition, it was taken into account that the stress of the regulation systems is also expressed in an increase in the activity of the sympathetic part of the vegetative nervous system. This part regulates the mechanism responsible for the mobilization of energy and metabolic resources under various loads. With a decrease in adaptive capacity, mobilization of the sympathetic nervous system plummets.

Thus, using the assessment of heart rate variability and the stress level of the regulation systems according to the RCG data, it is possible to accurately assess the level of body adaptation to the conditions of sports activity, as well as the effectiveness of any impact on the body of the long-distance runners, including that of sports training (Vesterinen et al., 2013; Dong, 2016; Novak, Saul, \& Eckberg, 1997). It was taken into account that rhythmocardiography is an image of consecutive rows of intersystolic intervals of an electrocardiogram in the form of straight line segments whose length is equivalent to the pause between heart contractions in seconds.

The study of heart rate variability in long-distance runners was carried out in the morning, at rest and fasting, and immediately after training in the preparatory period of the training cycle before competitions. The athlete was informed about the time and content of the diagnostic procedure, and provided consent to participate in the experiment. Before recording, a 10-minute rest was given to increase the stationarity of the process of RCG recording.

The study was carried out on a computer analyzer "Cardiometer-MT" by "Mikard Lana" LLP (State Register of Products - No. 98/219-67). The experiment used short 5-minute recordings made and analyzed in accordance with the International Standard (Novak et al., 1997; Rajendra Acharya et al., 2006) and current data on the features of heart rate variability in athletes (Pieralisi et al., 2017; Vesterinen et al., 2013; Aubert, Seps, \& Beckers, 2003).

The following RCG indicators most accurately proven in the study of athletes were used

- RRav. - the average interval between cardiocycles in ms;

- RRmin and RRmax - minimum and maximum interval between cardiocycles in $\mathrm{ms}$;

- $\mathrm{dX}$ - variation range - difference between the longest and shortest RR interval (cardiocycle) in milliseconds (ms);

- SDNN - standard deviation of the NN interval from the average value in $\mathrm{ms}$. It is calculated as the square root of the RR intervals spread. SDNN reflects all cyclic components responsible for variability during the recording period;

- CV (\%) (coefficient of variation) does not differ from SDNN in terms of physiology but is normalized by heart rate;

- RMSSD - square root of the mean squared differences of successive $\mathrm{NN}$ intervals in $\mathrm{ms}$.

- Mo - mode (ms) - the range of the most frequently occurring values of cardiointervals - the peak of the histogram. 
It shows the most likely (dominant) level of functioning of the sinus node. This is the highest RR interval - peak on the histogram.

- AMO (\%) mode amplitude - the percentage of cardiointervals that fall into the range of mode, in relation to all cardiointervals. Mode amplitude depends on the influence of the sympathetic part of the VNS and reflects the degree of centralization of the heart rhythm control.

Analysis of the wave structure of the heart rhythm

- TP - the total power (area of waves on the RCG) of the RCG wave spectrum in ms2;

- HF - fast or high-frequency oscillations of the RCG wave spectrum reflecting the work of the parasympathetic nervous system and the autonomous circuit for regulating the heart rhythm (frequency range from 0.15 to $0.4 \mathrm{~Hz}$ ) in $\mathrm{ms} 2$;

- LF - slow or low-frequency oscillations of the RCG wave spectrum reflecting the work of the sympathetic nervous system and the central rhythm control circuit (frequency range from 0.04 to $0.15 \mathrm{~Hz}$ ) $\mathrm{ms} 2$;

- VLF - very slow or very low-frequency oscillations of the wave spectrum of the RCG reflecting the work of the central and humoral channels of heart rhythm regulation (frequency range from 0.04 to $0.015 \mathrm{~Hz}$ ) ms2

\section{Indices by Baevsky R.M.}

- VRI (vegetative rhythm index) AMo/Mo $\times d X$. The smaller the VRI, the higher the activity of the parasympathetic part and the autonomous circuit.

- RPAI (regulatory processes adequacy indicator) AMo/ Mo to identify the relationship between the level of functi- oning of the sinus node and sympathetic activity. This is the indicator reflecting the interaction between the autonomous circuit and humoral regulation channel.

- SI (regulatory systems stress index) $\mathrm{AMo} / 2 \mathrm{dX} \times \mathrm{Mo}$ reflects the degree of centralization of heart rhythm control.

The training was monitored using FirstBeat SPORT software and hardware, which incorporates special BodyGuard sensor which allows the athlete's cardio signal to be recorded for up to 7 days and FirstBeat BodyGuard software by FirstBeat (Finland).

The software and hardware offer: analysis of the training process using pulsometry and heart rate variability, including - remote analysis, recovery monitoring after exercise, assessment and monitoring of the total stress to account for its after-training component.

The software converts information about pulsometry and rhythm variability into a unique digital model in accordance with advanced methods of mathematical data processing and provides specialized reports.

"FirstBeat SPORT" complex (www.firstbeat.com) is a single-channel electrocardiograph with a large built-in memory capacity that allows recording a pulsogram continuously for up to 7 days.

The exclusiveness of Firstbeat technology is that with the help of equipment it is possible to collect information about the state of the rhythm control circuit around the clock and, at the same time, measure training load and afterload recovery indicators in real time and present the results in graphical and digital forms that reflect the quality of training, daytime activity and night recovery.

Figure 1 presents data on the work of an athlete during training in various training zones in the form of a bar chart.
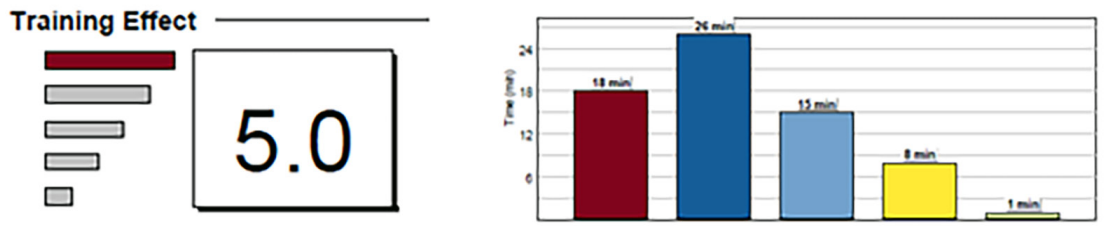

Figure 1. Data on training zones and training effect

Number 5 in the left part of the figure indicates the amount of the training effect (TE) on a scale from 1 to 5 (www.firstbeat.com). Explanation of the values in this figure is given in Table 1.

Table 1. Training Effect (TE)

\begin{tabular}{ll}
\hline TE ranges & Description \\
\hline $1.0-1.9$ & Daily activity \\
$2.0-2.9$ & Mild aerobic workout \\
$3.0-3.9$ & Medium intensity workout \\
$4.0-4.5$ & High intensity workout \\
$4.5-5.0$ & Workout with the signs of overtraining \\
\hline
\end{tabular}

The software calculates the TE automatically according to the amount of oxygen debt for each specific athlete, taking into account their height, weight, as well as the skill level.

The pulsogram and cardiointervalogram were recorded for athletes continuously at a training camp on the flat terrain and at the medium altitude with the help of the BodyGuard sensor. The data was processed on a personal computer using Firstbeat SPORT software. The analysis of the RCG records was performed in accordance with the 1996 International Standard and FirstBeat recommendations.

\section{Results}

The HRV analysis was carried out by means of 5-minute records at rest in the morning before breakfast and immediately after the training on the first day of the training session to study the dynamics of RCG indicators for long-distance runners (EG-1), who trained on the on the 
flat terrain, in comparison with the group (EG-2) which trained at the medium altitude. The average values of the training effect on this day in the two groups did not significantly different 3.5 \pm 0.17 (EG-1), versus $3.8 \pm 0.24 \mathrm{cu}$ (EG-2), ( $\mathrm{p}>0.05)$.
These rhythmograms after the workout were compared with the indicators obtained at rest. The median RCG indicators after training session (EG-2), in comparison with those obtained at rest, are presented below in Table 2 .

Table 2. Medians of RCG Indicators after Training in Comparison with those Obtained at Rest in Athletes from Groups EG-1 and EG-2

\begin{tabular}{|c|c|c|c|c|c|}
\hline \multirow{3}{*}{ Parameters } & \multicolumn{5}{|c|}{ Median RCG indicators of athletes before and after training } \\
\hline & \multicolumn{2}{|c|}{ At rest } & \multicolumn{2}{|c|}{ After training } & \multirow{2}{*}{$\mathbf{P}$} \\
\hline & EG-1 & EG-2 & EG-1 & EG-2 & \\
\hline Mo, MS & 1000.3 & 1050.0 & 740.5 & 600.1 & $>0.05$ \\
\hline Amo, \% & 36.1 & 24,1 & 48.3 & 72.1 & $<0.05$ \\
\hline $\mathrm{RR} \min , \mathrm{ms}$ & 863.0 & 772.1 & 665.8 & 568.6 & $>0.05$ \\
\hline RR max, ms & 1121.2 & 1352.2 & 933.2 & 650.2 & $>0.05$ \\
\hline $\mathrm{dX}, \mathrm{ms}$ & 258.0 & 0.4 & 267.4 & 81.6 & $<0.05$ \\
\hline $\mathrm{CV}, \%$ & 5.7 & 7.5 & 4.7 & 3.7 & $<0.05$ \\
\hline SDNN, ms & 56,1 & 81.0 & 40.6 & 33.0 & 0.01 \\
\hline RMSSD, ms & 53.0 & 77.0 & 35.9 & 13.2 & 0.02 \\
\hline VRI (cu) & 5.5 & 2.6 & 8.6 & 14.5 & $<0.05$ \\
\hline RPAI (cu) & 39.7 & 24.9 & 36.1 & 13.6 & $<0.05$ \\
\hline SI (cu) & 101.2 & 47.7 & 243.6 & 338.0 & 0.02 \\
\hline $\mathrm{HF}, \mathrm{ms} 2$ & 1126.4 & 2138.6 & 571.6 & 150.6 & 0.05 \\
\hline $\mathrm{LF}, \mathrm{ms} 2$ & 1531.6 & 1962.4 & 722.0 & 540.2 & $<0.01$ \\
\hline $\mathrm{VLF}, \mathrm{ms} 2$ & 552.6 & 1566.3 & 538.5 & 156.0 & 0.04 \\
\hline TP, ms2 & 3210.6 & 5666.3 & 1832.1 & 846.8 & $<0.05$ \\
\hline LF/HF (cu) & 1.4 & 0.9 & 1.3 & 3.6 & $>0.05$ \\
\hline LF, \% & 47.7 & 34,6 & 39,4 & 63.8 & $>0.05$ \\
\hline $\mathrm{HF}, \%$ & 35.1 & 37.7 & 31.2 & 17.8 & $>0.05$ \\
\hline
\end{tabular}

The study showed that the differences in the RCG indi- on of function at rest. On the contrary, after the load, the ces between the groups (EG-1) and (EG-2) turned out to be reliable after training for Amo, $\mathrm{dX}$, all complex and spectral indices of the heart rhythm, except for the high-frequency component of the heart rhythm (HF).

At rest, the RCG differences between (EG-1) and (EG-2) were mainly associated with lower rates of heart rate variability (EG-1) initially, which reflected a lower economizatirhythmograms (EG-1) were characterized by low rhythm centralization after load compared to group (EG-2), which indicated lower mobilization ability of athletes who train on the flat terrain.

The mobilization degree - changes in \% of rhythmogram indicators after a workout versus rest in each group of athletes are presented in Table 3.

Table 3. The Percentage of Changes in the Initial RCG Indicators in Athletes of the Two Groups after Training and the Ratio of the Extent of These Changes

\begin{tabular}{cccc}
\hline Parameter & EG-1 & EG-2 & Ratio EG-2/EG-1 \\
\hline Mo, ms & 26.0 & 42.8 & 1.7 \\
Amo, \% & -33.8 & -200.4 & 5.9 \\
RR min, ms & 22.9 & 26.3 & 1.2 \\
RR max, ms & 16.8 & 51.9 & 3.1 \\
dX, ms & -3.6 & 85.9 & 23.6 \\
CV, \% & 17.5 & 50.7 & 2.9 \\
SDNN, ms & 27.6 & 59.3 & 2.1 \\
RMSSD, ms & 32,3 & 82.9 & 2.6 \\
VRI (cu) & -56.4 & -457.7 & 8.1 \\
RPAI (cu) & 9.1 & 45.4 & 5.0 \\
SI (cu) & -140.7 & -608.6 & 4.3 \\
HF, ms2 & 49.3 & 93.0 & 1.9 \\
LF, ms2 & 52.9 & 72.5 & 1.4 \\
VLF, ms2 & 2.6 & 90.0 & 35.3 \\
TP, ms2 & 42.9 & 85.1 & 2.0 \\
LF/HF (cu) & 7.1 & -300.0 & 42.0 \\
LF, \% & 17.4 & -84.4 & 4.9 \\
HF, \% & 11.1 & 52.8 & 4.8 \\
\hline
\end{tabular}


Positive values in the table indicate the percentage of decline after exercise in relation to the baseline figures, and negative values reflect the percentage increase. The third column of the table shows how many times the changes in EG-2 exceed the changes in the RCG after training for EG-1 long-distance runners, which are also graphically reflected in Figure 2.

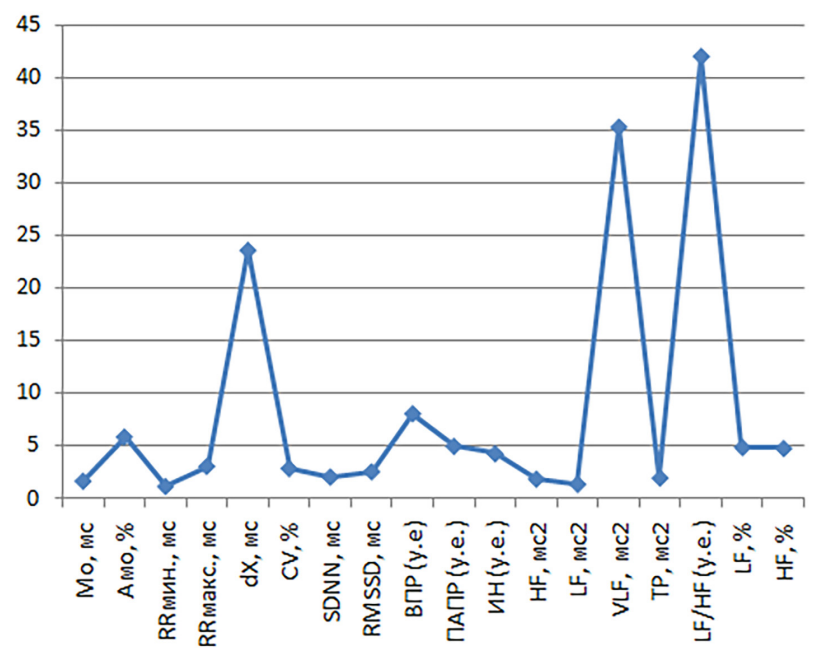

Figure 2. Multiplicity of changes in the RCG in terms of the rest/load ratio in EG-1 in comparison with the EG-2

As can be seen from Table 3 and Figure 2, the maximum difference in changes in EG-1/EG-2 after the load was noted in relation to the growth of $\mathrm{dX}, \mathrm{VRI}, \mathrm{VLF}$, and the maximum was observed for LF/HF (42-fold). This indicates a lower inclusion of the central control circuit during the load of the long-distance runners in EG-1 as compared to EG-2. It may be associated with the development of protective inhibition of the central nervous system in the long-distance runners from EG-1, low activation and mobilization of the body in response to the load. Such a low mobilization of the central control circuit in long-distance runners from EG-1 reflects the considerable overload of the central circuit at rest, since the functioning of the regulation system is evaluated by its response to the impact.

\section{Discussion}

It is known that the dynamics of rest/load indicators, as a rule, depends on the skill level of the athlete and the type of sport. More successful athletes have the highest level of sympathetic reaction and activity of the central circuit for the regulation of heart rhythm to the load as a manifestation of functional reserves mobilization (Bolotin, \& Bakayev, 2017c).

In this regard, it was interesting to analyze the initial types of regulation of the two groups of athletes. In EG-1 long-distance runners at rest, the central control circuit prevailed, which was cumulatively observed in $43.9 \%$ of EG-1 long-distance runners vs. $10.6 \%$ in EG-2, that is, four times more often with a high degree of differences confidence ( $\mathrm{p}$ $<0.001$ ). According to the data obtained, the majority of athletes had the optimal state of the body's regulatory systems, including their response to physical exertion. In aggregate, the types of regulation that are optimal for running were found in $56.1 \%$ of long-distance runners in EG-1 versus $89.5 \%$ of athletes in EG-2, that is, 1.6 times less often $(p<0.001)$.

Thus, the findings of a comprehensive assessment of heart rate variability in the evaluation of short 5-minute records and long (weekly) records indicate that, compared with EG2, long-distance runners from EG-1 adapt to the conditions of sports activity with higher strain on regulatory systems at rest. This is due to the lack of proper economization against the background of the initially low reserve and aerobic capabilities of the body, due, inter alia, to the low development of the autonomous control circuit.

One of the reasons behind such significant differences in HRV of the two groups of athletes is, apparently, that compensation in EG-1 long-distance runners is due to the strain of other regulatory systems of the body that help adapt to the conditions of sports activity. Secondly, low regulatory capabilities and fitness of long-distance runners in EG-1 are certainly lower than in the EG-2 athletes, which also increases the stress of adaptation mechanisms.

The above leads to overload and depreciation of the central control circuit at rest and, in connection with this, its low response to the impact under the conditions of physical and competitive loads. The cost of adaptation of such an organism increases significantly, its adaptation and competitive potential decreases. Accordingly, with a highly developed autonomous control circuit, we can expect a higher competitive success of the EG-2 long-distance runners compared to the EG-1 long-distance runners with poorly expressed control circuit.

Improvement in the process of arranging and expanding the content of training for long-distance runners using multidirectional means under the conditions of medium altitude aimed at developing stamina creates favorable conditions for the further development of the adaptive capabilities of the functional systems of their body. This contributed to the effectiveness of competitive activity of long-distance runners.

\section{Acknowledgements}

There are no acknowledgements.

\section{Conflict of Interest}

The authors declare that there are no conflicts of interest.

Received: 10 March 2019| Accepted: 10 May 2019|Published: 01 June 2019

\section{References}

Aubert, A.E., Seps, B., \& Beckers, F. (2003). Heart rate variability in athletes. Sports Medicine, 33(12), 889-919. https://doi.org/10.2165/00007256-20033312000003 
Bakaev, V.V., Bolotin, A.E., \& Vasil'eva, V.S. (2015). Factors determining sports specialization of cross country skiers. Teoriya i Praktika Fizicheskoy Kultury, 2, 40-41.

Bakaev, V.V., Bolotin, A.E., \& Aganov, S.S. (2016). Physical training complex application technology to prepare rescuers for highland operations. Teoriya Praktika Fizicheskoy Kultury, 6, 6-8.

Bakayev, V., Bolotin, A., \& You, C. (2018). Reaction of vegetative nervous system to loads in female long-distance runners with different fitness level. Journa of Human Sport and Exercise, 13(2), 245 - 252. doi: https://doi.org/10.14198/ jhse.2018.13.Proc2.09

Bohuslavska, V., Furman, Y., Pityn, M., Galan, Y., \& Nakonechnyi, I. (2017). Improvement of the physical preparedness of canoe oarsmen by applying different modes of training loads. Journal of Physical Education and Sport, 17(2), 797803. DOI:10.7752/jpes.2017.02121

Bolotin, A., \& Bakayev V. (2017a). The differences in response of the respiratory system of long and middle-distance runners and their influence on recovery rate. Journal of Physical Education and Sport, 17(4), 2443-2446. doi: 10.7752/jpes.2017.04272

Bolotin, A., \& Bakayev, V. (2017b). Method for Training of Long Distance Runners Taking into Account Bioenergetic Types of Energy Provision for Muscular Activity. In Proceedings of the 5th International Congress on Sport Sciences Research and Technology Support, 126-131.

Bolotin, A., \& Bakayev, V. (2017c). Peripheral circulation indicators in veteran trail runners. Journal of Physical Therapy Science, 29(6), 1092-1094. doi:10.1589/ jpts.29.1092

Bolotin, A., \& Bakayev, V. (2016). Factors that determine high efficiency in developing speed and strength abilities of female hurdlers. Journal of Physical Education and Sport, 16(3), 910-913. doi:10.7752/jpes.2016.03143
Bolotin, A., \& Bakayev, V. (2018). Pedagogical practice for development of coordination potential of MMA fighters and estimation of its efficiency. Journal of Human Sport and Exercise, 13(1), 72-88. doi:https://doi. org/10.14198/jhse.2018.131.08

Boullosa, D.A., Tuimil, J.L., Leicht, A.S., \& Crespo-Salgado, J.J. (2009). Parasympathetic modulation and running performance in distance runners. Journal of strength and conditioning research, 23(2), 626-631. doi:10.1519/JSC.0b013e31818dc44e

Dong, J. (2016). The role of heart rate variability in sports physiology (Review). Experimental and Therapeutic Medicine, 11, 1531-1536. https:// doi.org/10.3892/etm.2016.3104

Novak, V., Saul, J.P., \& Eckberg, D.L. (1997). Task Force report on heart rate variability. Circulation, 96(3), 1056-1057. PubMed PMID: 9264529

Pieralisi, M., Di Mattia, V., Petrini, V., De Leo, A., Manfredi, G., Russo, P., Scalise, L., \& Cerri, G. (2017). An Electromagnetic Sensor for the Autonomous Running of Visually Impaired and Blind Athletes (Part II: The Wearable Device). Sensors (Basel, Switzerland), 17(2), 381. doi:10.3390/ s17020381

Rajendra Acharya, U., Paul Joseph, K., Kannathal, N., Choo, M.L., \& Jasjit, S.S. (2006). Heart rate variability: a review. Medical \& Biological Engineering \& Computing, 44(12), 1031-1051. https://doi.org/10.1007/ s11517-006-0119-0

Vesterinen, V., Häkkinen, K., Hynynen, E., Mikkola, J., Hokka, L., \& Nummela, A. (2013). Heart rate variability in prediction of individual adaptation to endurance training in recreational endurance runners. Scandinavian Journal of Medicine \& Science in Sports, 23(2), 171-180. doi:10.1111/j.1600-0838.2011.01365.x 